\title{
Impact of structural uncertainties on the buckling strength of cylindrical GLARE panels subjected to uniform compression
}

\author{
Costas Kalfountzos ${ }^{1 *}$, George Bikakis ${ }^{1}$, and Efstathios Theotokoglou ${ }^{1}$ \\ ${ }^{1}$ National Technical University of Athens, Department of Mechanics, GR 15773 Zografou, Greece
}

\begin{abstract}
The objective of this article is the investigation of the elastic buckling strength of cylindrical simply supported GLARE (GLAss REinforced) panels subjected to axial compression using probabilistic analysis methods, so that the effect of uncertainties associated with material properties and dimensions of the panels on their elastic buckling load can be evaluated. The mechanical properties of aluminum along with the dimensions of aluminum and unidirectional (UD) glass-epoxy layers are considered to be random input variables whereas the critical buckling load is defined as a random output parameter. The employed eigenvalue buckling analysis and the probabilistic finite element analysis were carried out with ANSYS software. The Probabilistic Design System (PDS), along with the Monte Carlo Simulation and the Latin Hypercube Sampling method were used for the calculations. It is found that the thickness of aluminum layers has the strongest effect on the buckling strength, among the considered random input variables. It is also demonstrated that there is a considerable probability for the buckling load of GLARE panels to be overestimated when a deterministic analysis is conducted.
\end{abstract}

\section{Introduction}

Fiber Metal Laminates (FMLs) are hybrid composite materials, which were originally developed for application in primary aircraft structures. FMLs combine significant advantages of metals and fiber-reinforced composites. They demonstrate high fatigue resistance, high fracture toughness and excellent impact resistance which is comparable with the impact resistance of their constituent aluminum alloys. Low density, excellent moisture, corrosion and fire resistance are some other significant advantages of FMLs, which make them ideal for aerospace applications [1]. GLARE (GLAss REinforced) is the most successful FML up to now, since it has been applied in the production of primary aircraft structures, such as the fuselage of the Airbus A380 [2].

The unstiffened cylindrical panels are employed in a large number of structures subjected to high compressive loads which are prone to buckling. Furthermore, the calculation of elastic buckling load of the unstiffened panels is necessary for the consideration of the local buckling modes in the analysis of the, more widely used, stringer-

* Corresponding author: koskalf@yahoo.gr 
stiffened cylindrical shells [3]. Consequently, since FMLs are mainly used for the construction of thin-walled aerospace structures, the buckling strength of unstiffened FML panels is of great practical importance. The buckling strength of a structural component is affected by its exact dimensions and the materials used. However, due to variations in the raw material and the processes used, mechanical properties of a structural component vary [4-6]. Additionally, the variability in the manufacturing process because of unavoidable practical imperfections leads to undesirable deviations of the actual from the nominal dimensions [5]. These factors motivated the objective of this study, which is the investigation of the buckling strength of cylindrical simply supported GLARE panels subjected to axial compression using probabilistic analysis methods, so that the effect of uncertainties associated with material properties and dimensions of the panels on their elastic buckling load can be evaluated.

\section{Problem definition}

A thin circular cylindrical GLARE panel is considered under uniform axial compression. GLARE consists of thin 2024-T3 aluminum sheets bonded into one laminate with intermediate unidirectional (UD) glass-epoxy layers. The panel has length $L$, radius of curvature $R$, width $b<L$ and panel angle $\varphi$. The thickness $t$ of the panel is very small in comparison with its length and width, and the $R / t$ ratio is always greater than 165 yielding a shallow panel since it is well above the pertinent limit of $R / t=50$ [7]. The panel is loaded statically with a uniaxial constant compressive load $N_{z}$ along the curved edges. It will buckle for a specific value of the applied load since it is allowed to deform axially. Two different types of simply supported boundary conditions (bc) are considered and are described in section 3 of this article.

The classical eigenvalue buckling analysis of ANSYS FEM software [8] is employed in order for the critical buckling load $N_{z c}$ to be calculated numerically. The probabilistic elastic buckling behavior of two GLARE grades is investigated using the Probabilistic Design System (PDS) of ANSYS. The critical buckling load of each panel is defined as the response parameter which is a random function of the panel's variable material properties and dimensions.

\section{Finite element modeling procedure}

In this article, we carry out a three-dimensional FEM procedure in order to calculate the critical buckling load of the considered panels subjected to uniform axial compressive loading. SHELL 181 elements have been selected for the numerical simulation. These elements have four nodes with three translational and three rotational degrees of freedom and are suitable for analyzing thin layered shell structures [8], such as the thin panels of this study. The material behavior of 2024-T3 aluminum and composite plies is idealized using linear elastic material models with isotropic and orthotropic material properties, respectively. The uniaxial compressive stress $\sigma_{z}$ is simulated by a line pressure load $N_{z}$, which is always equal to unity, and is applied along the two curved edges of the panels.

The calculation of the critical buckling load $N_{z c}$ is accomplished using the classical eigenvalue buckling analysis of ANSYS [8] and the convergence of FEM results is always verified by comparison of results corresponding to fine and very fine panel mesh density. Additionally, the stress levels of the plies corresponding to the critical buckling load of the panel are always checked, in order to ensure that elastic buckling occurs. Apart from the bc, the curvature parameter of the GLARE panels is considered variable in order to study their buckling behavior using probabilistic analysis. 
The curvature parameter $Z$ of laminated panels is calculated by [7]:

$$
Z=\frac{b^{2}}{R} \sqrt{\frac{A_{11} A_{22}-A_{12}^{2}}{12 \sqrt{A_{11} A_{22} D_{11} D_{22}}}}
$$

where $A_{i j}$ and $D_{i j}(i, j=1,2)$ are the extensional and bending stiffnesses of the laminate.

The length and the thickness of the panels are constant: $L=1 \mathrm{~m}$ and $t=1.875 \mathrm{~mm}$, whereas the width $b$ and the radius of curvature $R$ of the panels vary: $0.5 m \leq b \leq 0.8 m$ and $0.31 \mathrm{~m} \leq R \leq 12 \mathrm{~m}$ so that the curvature parameter is approximately greater than 10 and lower than 1000 . The range of $Z$ between 10 and 1000 for isotropic panels is characterized by a transition from flat plate to complete cylinder behavior [7, 9].

Two different types of simply supported bc are considered for the panels, namely CSS (Classically Simply Supported) and CCSLS (Curved edges Classically Simply supported and Longitudinal edges Simply supported). The applied CSS bc are analyzed by Timoshenko \& Gere [10]: the radial displacements become zero along the edges of the panel, the axial displacements become zero along the straight edges and the circumferential displacements become zero along the curved edges. The CCSLS bc differs from CSS bc only at the axial displacements along the straight edges which are not constrained [3].

The probabilistic finite element analysis is applied to the following GLARE grades: GLARE 3-3/2-0.458, [Al $\left./ 0^{\circ} \mathrm{gl} / 90^{\circ} \mathrm{gl} / \mathrm{Al} / 90^{\circ} \mathrm{gl} / 0^{\circ} \mathrm{gl} / \mathrm{Al}\right]$

GLARE 5-3/2-0.292, $\left[\mathrm{Al} / 0^{\circ} \mathrm{gl} / 90^{\circ} \mathrm{gl} / 90^{\circ} \mathrm{gl} / 0^{\circ} \mathrm{gl} / \mathrm{Al} / 0^{\circ} \mathrm{gl} / 90^{\circ} \mathrm{gl} / 90^{\circ} \mathrm{gl} / 0^{\circ} \mathrm{gl} / \mathrm{Al}\right]$

The $0^{\circ}$ fiber orientation angle is parallel with the length $L$. The last number of the aforementioned coded name of GLARE panels indicates the thickness of aluminum layers in mm. Each composite ply of the examined GLARE laminates consists of S2-glass UD fiber prepreg and has a nominal thickness of $0.125 \mathrm{~mm}$. The material properties of aluminum and S2-glass UD prepreg layers considered for our calculations can be found in reference [11].

The FEM procedure and the suitability of the applied CSS bc of this study have been validated with comparisons of FEM results versus corresponding theoretical results for monolithic aluminum, composite and GLARE panels, as it is described in reference [12]. A very good agreement between the numerical and the theoretical results for all considered material systems has been found in [12].

\section{Probabilistic analysis}

The mechanical properties of a specific material vary because of unavoidable variations in the raw material and the applied processes. Similarly, the actual dimensions of a structural component will differ from the nominal values due to uncontrollable variability in the manufacturing process. These deviations from the nominal values are constrained by strict manufacturing tolerances, such as ISO tolerance system [13]. The real concern to take into account these random variations in order to design reliable FML structures makes the use of probabilistic analysis methods mandatory.

Taking into account that aluminum governs the analyzed linear elastic buckling response of GLARE panels [12] and that only the Young's modulus of aluminum is a practically uncertain mechanical property $[14,15]$, we define the aluminum 2024-T3 Young's modulus as a random input variable. Furthermore, the thicknesses of aluminum and UD glass-epoxy layers, the length and the width of the panel are considered to be random input variables as well, whereas the critical buckling load is defined as a random output parameter.

The probabilistic finite element analysis was performed using the PDS of ANSYS software [8]. Normal (Gaussian) distribution functions are considered for the five 
aforementioned random input variables. It is a common practice to consider that the mechanical properties and dimensions of components are normally distributed $[5,8,16]$. The Monte Carlo Simulation (MCS) was selected along with Latin Hypercube Sampling method. The convergence of PDS results is achieved by running a large number of simulation loops, for each panel, and is always verified in combination with additional ANSYS recommendations. GLARE 3 and GLARE 5 panels with CSS and CCSLS bc were studied using the PDS. In reference [14] the compression properties of 2024-T3 aluminum were evaluated experimentally and the mean value of Young's modulus was found to be $73.87 \mathrm{GPa}$ with a standard deviation of $1.88 \mathrm{GPa}$. Since the mean value of $73.87 \mathrm{GPa}$ practically coincides with reference [6], we consider the experiments of [14] reliable and employ the aforementioned mean and standard deviation values of Young's modulus in our calculations.

Taking the manufacturing process of the FMLs into account [17], it is reasonable to consider that the tolerances concerning the width and the length of the panel are equal to the corresponding tolerances of the aluminum layers. In our probabilistic analysis these tolerances along with the thickness tolerance of 2024-T3 aluminum layers are taken from industrial data sheets concerning 2024-T3 aluminum alloy [18]. The thickness tolerance of $0.125 \mathrm{~mm}$ thick UD glass-epoxy composite layers is given in reference [4] and we employ the same thickness tolerance for our calculations, since the UD glass-epoxy layers of the GLARE 3-3/2-0.458 and GLARE 5-3/2-0.292 panels are also $0.125 \mathrm{~mm}$ thick. For the definition of the normal distributions of the four random dimensions of the panels, each nominal dimension is considered equal to the mean value $\omega$ with a standard deviation $\sigma_{d}$ equal to tolerance / 3 . This implies that with probability 0.997 , each random dimension of the panel is within $\omega \pm 3 \sigma_{d}$ [5]. As an example, the tolerances of these four random input variables are shown in Table 1 for a specific GLARE 3-3/2-0.458 panel.

Table 1. Tolerances $[4,18]$ of the dimensions of a GLARE 3-3/2-0.458 cylindrical panel.

\begin{tabular}{|c|c|c|}
\hline GLARE 3-3/2-0.458 & Nominal dimension [mm] & Tolerance [mm] \\
\hline Aluminum layer thickness & 0.458 & \pm 0.0381 \\
\hline Glass-epoxy layer thickness & 0.125 & \pm 0.005 \\
\hline Width of the panel & 750 & \pm 3.175 \\
\hline Length of the panel & 1000 & \pm 2.381 \\
\hline
\end{tabular}

Representative histograms of aluminum Young's modulus $E_{a l}$ and thickness of aluminum layers $t_{a l}$ are depicted in Fig. 1. It is shown for both input variables, that the histogram bars are close to the normal distribution curve and they are smooth without any major gaps, indicating that the number of simulations is sufficient. Additionally, the skewness and excess kurtosis of $E_{a l}$ and $t_{a l}$ shown in Fig. 1 are practically equal to zero, as it is expected for a normal distribution function. 
a
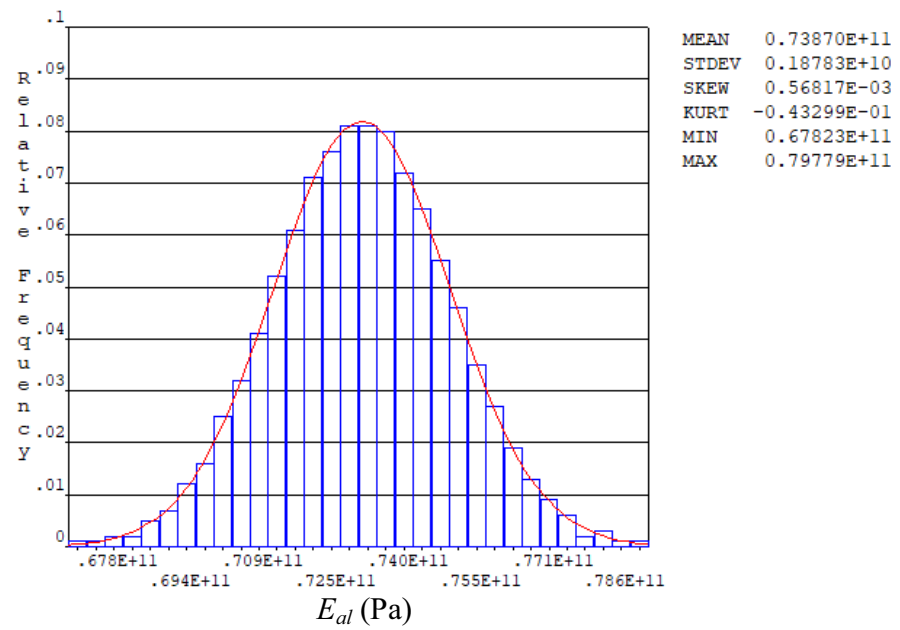

b

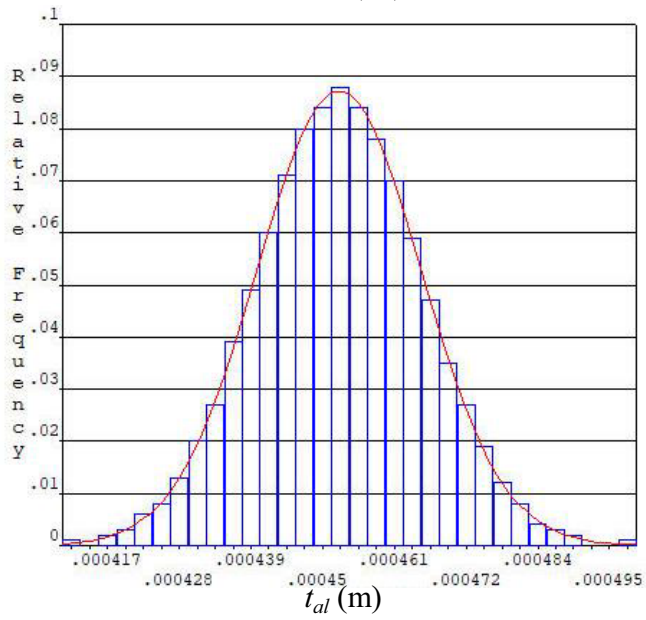

MEAN $\quad 0.45834 \mathrm{E}-03$

STDEV $\quad 0.12704 \mathrm{E}-04$

SKEW $\quad 0.76981 \mathrm{E}-02$

KURT $-0.53049 \mathrm{E}-02$

MIN $\quad 0.41697 \mathrm{E}-03$

MAX $0.50294 \mathrm{E}-03$

Fig. 1. Histogram (blue color) with values of statistical measures and normal distribution function (red color) of (a) aluminum Young's modulus and (b) thickness of $0.458 \mathrm{~mm}$ thick aluminum layers.

\section{Results and discussion}

Fig. 2 illustrates a representative histogram of $N_{z c}$. The indicated standard deviation is relatively low yielding that $N_{z c}$ values tend to be close to their mean value. The indicated excess kurtosis is almost equal to zero, a fact which means that the distribution of $N_{z c}$ is mesokurtic [8]. Referring to the skewness indicated in Fig. 2, it has a low positive value, a fact which indicates that the distribution of $N_{z c}$ has low asymmetry with a longer right tail. It is noted that the findings described in this paragraph are also valid for the $N_{z c}$ histograms of all GLARE 5 and GLARE 3 panels of our probabilistic analysis, regardless of their bc. 


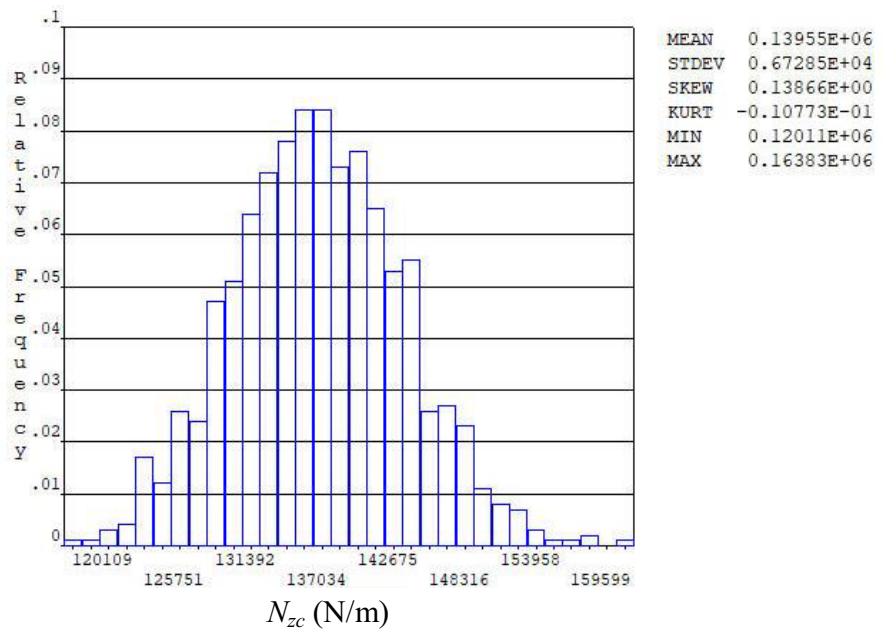

Fig. 2. Histogram with values of statistical measures of the critical buckling load for GLARE 3-3/20.458 cylindrical panel with CCSLS bc under uniform axial compression $(\mathrm{L}=1 \mathrm{~m}, \mathrm{~b}=0.75 \mathrm{~m}, \mathrm{R}=0.8 \mathrm{~m})$.

Using the PDS and the aforementioned uncertainties, which are realistic for practical applications, we found that there is a considerable probability to overestimate the critical buckling load of the panels if these uncertainties are ignored. For example, a CCSLS GLARE 3-3/2-0.458 panel $(L=1 m, b=0.75 m, R=0.8 m)$ has $19.9 \%$ probability for the critical buckling load to be $4 \%$ lower than it is predicted from deterministic analysis. Moreover, there is $4.4 \%$ probability for the critical buckling load to be $8 \%$ lower than the corresponding value from deterministic analysis.

The Spearman rank-order correlation coefficients $\left(r_{s}\right)$ between each one of the random input variables and the critical buckling load have been calculated and are presented in Table 2 for a GLARE 3 panel with CCSLS bc. The values of Table 2 represent the trend of $r_{s}$ for both GLARE grades studied in this article regardless of their bc. It is noted that $r_{s}$ is constrained as follows: $-1 \leq r_{s} \leq 1$ [19]. The value of +1 indicates that there is a perfect positive monotonic correlation between the two considered variables, whereas the value of -1 indicates that there is a negative one. When the value of $r_{s}$ is equal to zero there is not monotonic correlation between the two variables.

Table 2. Spearman rank-order correlation coefficients between random input variables and critical buckling load $\left(N_{z c}\right)$ for GLARE 3-3/2-0.458 cylindrical panel with CCSLS bc under uniform axial compression $(\mathrm{L}=1 \mathrm{~m}, \mathrm{~b}=0.7 \mathrm{~m}, \mathrm{R}=1.7 \mathrm{~m})$.

\begin{tabular}{|c|c|c|c|c|c|}
\hline & $\boldsymbol{E}_{\boldsymbol{a l}}$ & $\boldsymbol{t}_{\boldsymbol{a l}}$ & $\boldsymbol{t}_{\boldsymbol{c}}$ & $\boldsymbol{b}$ & $\boldsymbol{L}$ \\
\hline $\boldsymbol{N}_{z \boldsymbol{c}}$ correlation coefficients & 0.410 & 0.887 & 0.074 & -0.030 & -0.010 \\
\hline Significant correlation coefficient & yes & yes & yes & no & no \\
\hline
\end{tabular}

It is shown from Table 2 that the correlation coefficients are separated to significant and insignificant. More specifically, the hypothesis that the correlation coefficient between the elastic buckling load and a particular random input variable is identical to zero is tested and then the probability that this hypothesis is true is calculated. If this probability exceeds the significance level of $2.5 \%$ [8] the specific correlation coefficient is considered as insignificant. Among the significant ones, the thickness of aluminum layers has by far the highest value of $r_{s}$ indicating that there is a very strong positive monotonic correlation between this input variable and the elastic buckling load of the cylindrical panel. This finding is also validated from the scatter plot of Fig. 3(a), in which the positive correlation is clearly visualized and it is shown that the scatter of the values of $N_{z c}$ is relatively limited 
with respect to the plotted trendline. Aluminum Young's modulus follows with reduced value of $r_{s}$, which indicates that it has a moderate positive monotonic correlation with $N_{z c}$ and the thickness of S2/glass-epoxy layers $\left(t_{c}\right)$ has a very weak positive monotonic correlation with $N_{z c}$, since the value of $r_{s}$ now is close to zero.

The width and the length of the panel have the lowest absolute values of $r_{s}$ and they are considered as insignificant. Fig. 3(b) illustrates a scatter plot of the critical buckling load versus the length for a GLARE 3 panel and it indicates that there is not monotonic correlation between the two variables. There is a large scatter of the output parameter with respect to the plotted trendline. It can be observed from Fig. 3(b) that the coefficient of determination $R^{2}$ is practically equal to zero, which indicates that the trendline is not reliable.

a

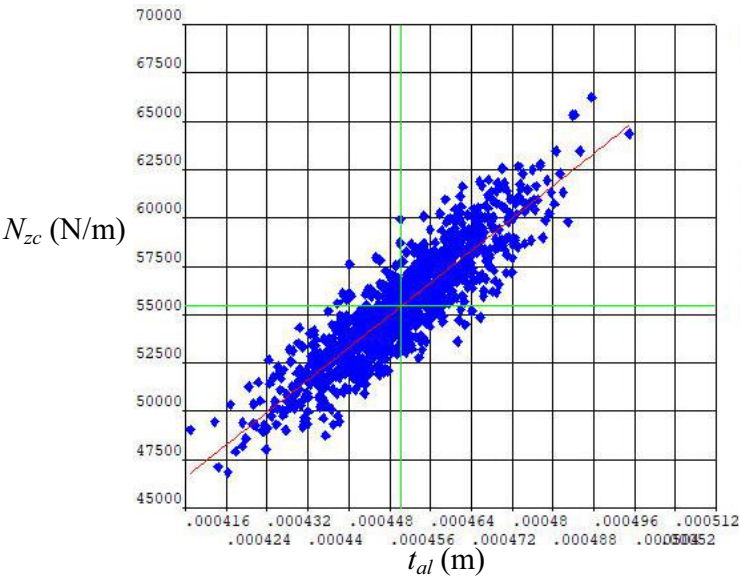

Rank-order Correlat. Coefficient 0.8871

inear Correlation Coefficient 0.8914

Trendline Type POLY: Co -40471

C1 .209E+09

Trendline Accuracy: CHI $0.2051 \mathrm{E}+03$ R2 $0.7945 \mathrm{E}+00$

b

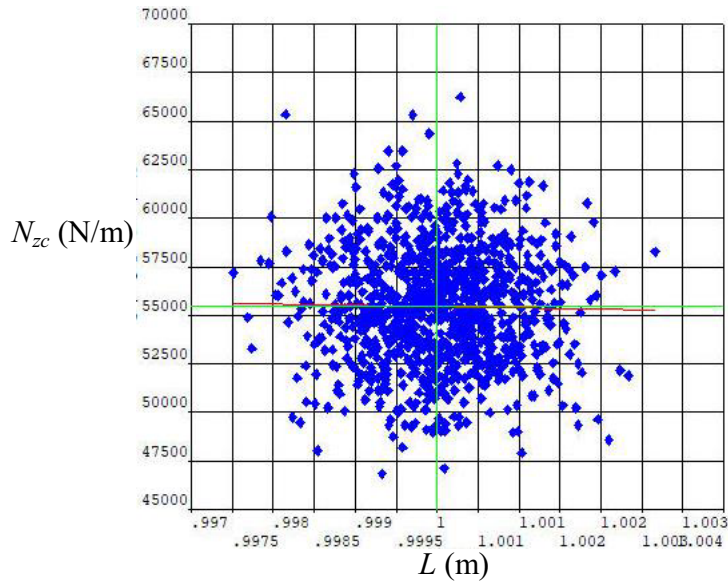

Rank-Order Correlat. Coefficient -0.0100

Linear Correlation Coefficient -0.0174 Trendline Type POLY: co 121017 C1 -65571

Trendline Accuracy: $\mathrm{CHI} 20.9987 \mathrm{E}+03$ R2 $-0.6974 \mathrm{E}-03$

Fig. 3. Scatter plots of the critical buckling load versus (a) thickness of aluminum layers and (b) panel length for GLARE 3-3/2-0.458 cylindrical panel with CCSLS bc under uniform axial compression $(\mathrm{L}=1 \mathrm{~m}, \mathrm{~b}=0.7 \mathrm{~m}, \mathrm{R}=1.7 \mathrm{~m})$.

\section{Conclusions}

This article deals with the investigation of the buckling strength of cylindrical GLARE panels, with two different types of simply supported bc, subjected to uniaxial compression using probabilistic analysis methods, so that the effect of uncertainties associated with the 
material properties and the dimensions of the panels on their elastic buckling load can be evaluated.

It is found that the thickness of aluminum layers has the strongest effect on the buckling strength, among the considered random input variables. More specifically, there is a very strong positive monotonic correlation between the thickness of aluminum layers and the buckling load. It is also found that there is a considerable probability for the buckling load of GLARE panels to be overestimated when only the deterministic values of mechanical properties and dimensions are considered.

\section{References}

1. T. Sinmazçelik, E. Avcu, M.O. Bora, O. Çoban, Mater. Des. 32, 3671-3685 (2011)

2. R.C. Alderliesten, R. Benedictus, J. Aircraft 45, 1182-1189 (2008)

3. L.H. Sobel, T. Weller, B.L. Agarwal, Comput. Struct. 6, 29-35 (1976)

4. C. Jiang, X. Han, G.P. Liu, Comput. Struct. 86, 1696-1703 (2008)

5. W.R. Blischke, D.N.P. Murthy, Reliability modeling, prediction and optimization, John Wiley \& Sons (2000)

6. R.C. Rice, J.L. Jackson, J. Bakuckas, S. Thompson, Metallic Materials Properties Development and Standardization (MMPDS), Office of Aviation Research, U.S. Development of Transportation (2003)

7. J.L. Verolme, The development of a design tool for fiber metal laminate compression panels, Ph.D. Dissertation, Delft University of Technology, Netherlands (1995)

8. ANSYS, Structural Analysis Guide, ANSYS Inc. (2014)

9. G. Gerard, H. Becker, Handbook of structural stability part III-buckling of curved plates and shells, TN-3783, NACA, USA (1957)

10. S.P. Timoshenko, J.M. Gere, Theory of elastic stability, Mc-Graw Hill, $2^{\text {nd }}$ ed. (1961)

11. A.S. Yaghoubi, B. Liaw, Int. J. Impact Eng. 54, 138-148 (2013)

12. C.D. Kalfountzos, G.S.E. Bikakis, E.E. Theotokoglou, Buckling of simply supported GLARE cylindrical panels subjected to uniform compression, in Proceedings of the $6^{\text {th }}$ International Virtual Conference of Engineering Against Failure, ICEAF VI, 23-25 June 2021 (2021)

13. ISO 2768-1, Tolerances for linear and angular dimensions without individual tolerance indications (1989)

14. R. Patel, Investigating the mechanical behavior of conventionally processed high strength aluminum alloy 2024, MSc Thesis, University of Akron, USA (2018)

15. P.E. Hess, D. Bruchman, I.A. Assakkaf, B.M. Ayyub, Nav. Eng. J. 114, 139-166 (2002)

16. B. Kriegesmann, E.L. Jansen, R. Rolfes, Compos. Struct. 94, 654-663 (2012)

17. J. Sinke, Manufacturing principles for fiber metal laminates, in Proceedings of the International Committee on Composite Materials (ICCM-17), 27-31 July 2009, Edinburgh, UK (2009)

18. Kaiser Aluminum, Sheet \& plate product capabilities, https://online.kaiser aluminum.com/depot/PublicProductInformation/Document/1010/Kaiser_Aluminum_S heet Plate.pdf (accessed 13 November 2020)

19. M.K. Chung, Correlation coefficient, N.J. Salkind (Ed.), Encyclopedia of Measurement and Statistics, Vol. 1, SAGE Publications (2007) 